\section{P36 LOSS OF POU4F2/BRN3B IN THE STRESSED HEART COMPROMISES THE ADAPTIVE HYPERTROPHIC RESPONSE IN MALE BRN3B KO MICE, BUT NOT BRN3B KO FEMALE MICE}

${ }^{1}$ Lauren Maskell, ${ }^{2}$ Daniel Stuckey, ${ }^{1}$ Vishwanie Budhram-Mahadeo. ${ }^{1}$ Rayne Building, 5 University Street, London; ${ }^{2}$ Paul O Gorman Building, 72 Huntley Street, London

\subsection{6/heartjnl-2018-BSCR.41}

Rationale A key feature of cardiovascular disease is cardiac hypertrophy, whereby terminally differentiated cardiomyocytes enlarge in response to stress. Hypertrophy can be beneficial to cardiac function (e.g., exercise or pregnancy); however pathological hypertrophy (e.g. chronic hypertension) is detrimental and leads to heart failure. The fate of cardiomyocytes during hypertrophy are dependent gene expression changes. Hence, cardiac transcription factors that control such changes by regulating target genes will be important for facilitating hypertrophy. The transcription factor POU $4 F 2 / B r n 3 b$ is highly expressed in the foetal heart and reduced in adult cardiomyocytes but is re-expressed in response to injury. Its interaction with other proteins such as the oestrogen receptor differentially regulate transcriptional activity; suggesting a difference in adaptation to physiological and pathological stress in male and female mice.

Methods and results Male and female Brn3b-KO mutant mice and wild type controls were used to assess the role of this TF in physiological (exercise on running wheels) and pathological stress (Angiotensin II infusion). Changes in cardiac function (cardiac output, etc.) were analysed using echocardiography/ ultrasound, histological changes were assessed (e.g. Masson's Trichrome) and genetic changes (e.g. qPCR, Western blot) were measured at baseline and 4 weeks post treatment. Following pathological and physiological stress, cardiac function and contractility were reduced in male Brn $3 \mathrm{~b}-\mathrm{KO}$ mice compared to baseline measurements. Exercise performance in Brn3b KO male mice was also reduced compared to WT mice. In contrast, cardiac function and contractility were not compromised in Brn3b KO female mice following physiological and pathological stress compared to baseline measurements. In addition, exercise performance in Brn $3 \mathrm{~b} \mathrm{KO}$ female mice was better when compared to WT mice.

Conclusion Brn3b may be important for the adaptive hypertrophic response in male mice, but not necessary for female mice, suggesting complex roles for Brn3b in the stressed heart.

\section{P37 THE ASSESSMENT OF THE CARDIOPROTECTIVE PROPERTIES OF METFORMIN DURING SUNITINIB- INDUCED CYTOTOXICITY}

Refik Kuburas, Mayel Gharanei, Irmgard Haussmann, Helen Maddock, Hardip Sandhu. Centre for Applied Biological and Exercise Sciences (ABES), Faculty of Health and Life Sciences, Coventry University, Coventry, UK

\subsection{6/heartjnl-2018-BSCR.42}

Multi-tyrosine kinase inhibitor Sunitinib is associated with cardiotoxicity, in contrast the anti-type- 2 diabetic Metformin is associated with cardioprotective properties via adenosine monophosphate-activated protein kinase (AMPK) activation.

We investigated whether treatment of rat langendorff hearts with Metformin attenuated Sunitinib-induced cytotoxicity via
AMPK. We further investigated the cotreatment of Metformin with Sunitinib using HepG2 and HL60 cancer cell lines to reveal if potential adjunctive therapy drugs interfere with and prevent the anti-cancer activity of chemotherapy agents.

Male Sprague-Dawley rat hearts were Langendorff perfused with vehicle or, Sunitinib $(1 \mu \mathrm{M})$ in the absence or presence \pm Metformin $(50 \mu \mathrm{M}) \pm$ S-4-Nitro-benzyl-theonosine (AMPK inhibitor, NBTI) $(1 \mu \mathrm{M})$ for $155 \mathrm{~min}$. Functional parameters were measured throughout the experiment $(n=6)$, heart tissue was also assessed for necrosis or relevant protein phosphorylation $(n=6)$.

HepG2 and HL60 cells treated in the presence of Sunitinib $(0.1-100 \mu \mathrm{M}) \pm$ Metformin $(50 / 1000 \mu \mathrm{M}) \pm$ NBTI $(1 \mu \mathrm{M}) \quad(\mathrm{n}=6)$ were assessed for cell viability..

Sunitinib caused a significant increase in myocardial infarcted tissue (Vehicle $=11 \% \pm 1 \%$ vs Sunitinib $=31 \pm 2 \%$, $\mathrm{p}<0.05)$. Metformin co-administration restored viable myocardial tissue (Sunitinib+Metformin $=20 \% \pm 2 \%, p<0.05$ ), NBTI attenuated Metformin's cardioprotection (Sunitinib+Metformin $+\mathrm{NBTI}=30 \% \pm 2 \%, \mathrm{p}<0.05)$.

Sunitinib decreased cell viability in HepG2 and HL60 cells at doses 5-100 $\mu \mathrm{M}$ (HepG2 IC50 $=21 \mu \mathrm{M}$, HL60 IC50=10 $\mu \mathrm{M})$. Cytotoxicity of Sunitinib was maintained in presence of $0.05 \mathrm{mM}$ Metformin (HepG2 IC50=76 $\mu \mathrm{M}$, HL60 IC50=22 $\mu \mathrm{M}$ ) and $1 \mathrm{mM}$ Metformin (HepG2 IC50=44 $\mu \mathrm{M}$, HL60 IC50 $=24 \mu \mathrm{M})$.

Metformin $(30-1000 \mu \mathrm{M})$ did not reduce HepG2 and HL60 cell viability.

This study shows for the first time that Metformin protects the hearts against Sunitinib-induced cardiotoxicity via AMPK activation which was attenuated with NBTI.

\section{P38 NLRP3 INFLAMMASOME PATHWAYS IN HUMAN ENDOTHELIAL CELLS} $1,2 \mathrm{R}$ Abas, ${ }^{1} \mathrm{M}$ Wozniak, ${ }^{1} \mathrm{KE}$ Herbert. ${ }^{1}$ Department of Cardiovascular Sciences, University of
Leicester, Leicester, UK; ${ }^{2}$ Department of Human Anatomy, Faculty of Medicine, Universiti
Putra Malaysia, Selangor, Malaysia

10.1136/heartjnl-2018-BSCR.43

Endothelial cells play roles in regulating blood flow and in control of inflammation, both of which play important roles in atherogenesis. It is known that the NLRP3 inflammasome is involved in innate defence mechanisms in monocytes and macrophages and requires two steps for activation, signal 1 and signal 2, which result in the production of active IL-1 $\beta$ and IL-18. The aim of this study was to investigate NLRP3 inflammasome activation in human endothelial cells. Phorbol 12-myristate 13-acetate (PMA; $5 \mathrm{ng} / \mathrm{ml}$ ) differentiated THP-1 cells (positive control), human umbilical vein endothelial cells (HUVEC) and EA.hy926 cells were 'primed' for signal 1 with lipopolysaccharide (LPS) $(0.1-5 \mu \mathrm{g} / \mathrm{ml})$ for 24 hours and then activated (signal 2) by exposure to ATP $(300 \mu \mathrm{M})$ for 1 hour. Using Western blotting, NLRP3 protein, pro-IL-1 $\beta$, pro-IL-18, active IL-1 $\beta$ and active IL-18 were observed in THP-1 cells demonstrating priming and activation of the inflammasome as expected. EA.hy926 cells but not HUVEC expressed pro-IL13. Inflammasome activation was confirmed by ELISA which detected any active end products in the cell culture supernatant. THP-1 cells secreted IL-1 $\beta$ and IL-18 as expected. However, for endothelial cells, only EA.hy926 cells showed some low level expression of active IL-1 $\beta$. In conclusion, although 
there was evidence of NLRP3 inflammasome priming for both endothelial cell types, only EA.hy926 cells showed extracellular IL-1 $\beta$ production. Other end products of NLRP3 inflammasome activation may be worthy of investigation in order to fully describe the response of endothelial cells to innate stimuli.

\section{P39 POST-MYOCARDIAL INFARCTION EXTRACELLULAR MATRIX REMODELLING IN THE PIG IS ASSOCIATED WITH ALTERED EXPRESSION OF MICRO-RNAS}

A Harvard, H Dobrzynski, AJ Atkinson, A D'Souza, J Yanni, M Petkova, B Borbas, N Malik, CM Holt. Division of Cardiovascular Sciences, University of Manchester

\subsection{6/heartjnl-2018-BSCR.44}

Extracellular matrix remodelling is a key component of myocardial repair after ischaemic injury, and contributes to fibrotic changes leading to heart failure. MicroRNAs, short non-coding RNAs which regulate gene expression, have been shown to be dysregulated in a number of rodent models of ischaemia and heart failure, yet limited data is available in large translational animal models.
The expression of a number of fibrosis-associated microRNAs were investigated in a relevant porcine model of mild to moderate left ventricular (LV) dysfunction induced by coronary microembolisation ( $n=3$ MI and $n=3$ control pigs). Samples of myocardial tissue were obtained 4 weeks post-MI and classified into infarct, border and remote (normal) regions. The infarct area was histologically mostly acellular with dense inflammatory infiltrate, extracellular matrix expansion and collagen deposition as seen with Masson's trichrome and Picrosirius red staining. qRT-PCR analysis of microRNA expression demonstrated downregulation of miR-133a $(p=0.0085)$, whereas miR-214 was upregulated $(p=0.0027)$ in the infarct region, compared to normal/control myocardium.

miR-133a has previously been shown to protect against cardiac fibrosis, and is downregulated in the heart in numerous small-animal models of heart failure. miR-214 is upregulated in the fibrotic heart, and its inhibition has been shown to be protective against hepatic fibrosis. Therefore, our porcine data supports the hypothesis that pathological myocardial remodelling may be influenced by altered expression of microRNAs, and confirms the pattern of microRNA expression observed in other species. Future work is aimed at testing therapeutic approaches targeting these specific microRNAs. 